\title{
Noise in Bose Josephson junctions: decoherence and phase relaxation
}

\author{
G. Ferrini,, , D. Spehner, ${ }^{1,2}$ A. Minguzzi, ${ }^{1}$ and F.W.J. Hekking ${ }^{1}$ \\ ${ }^{1}$ Laboratoire de Physique et Modélisation des Milieux Condensés, \\ Université Joseph Fourier and CNRS, B.P. 166, 38042 Grenoble, France \\ ${ }^{2}$ Institut Fourier and CNRS, Université Joseph Fourier, B.P. 74, 38402 Saint Martin d'Hères, France
}

\begin{abstract}
Squeezed states and macroscopic superpositions of coherent states have been predicted to be generated dynamically in Bose Josephson junctions. We solve exactly the quantum dynamics of such a junction in the presence of a classical noise coupled to the population-imbalance number operator (phase noise), accounting for e.g. the experimentally relevant fluctuations of the magnetic field. We calculate the correction to the decay of the visibility induced by the noise in the non-markovian regime. Furthermore, we predict that such a noise induces an anomalous rate of decoherence among the components of the macroscopic superpositions, which is independent on the total number of atoms, leading to potential interferometric applications.
\end{abstract}

PACS numbers: 03.75.Gg,03.75.Mn

\section{INTRODUCTION}

It has been realized in the last decade that an ultracold Bose gas trapped in an optical potential offers the possibility to manipulate coherently entangled many-body quantum states, with interesting applications in precision measurements and in quantum information. For instance, spin squeezed states and macroscopic superpositions of coherent states are generated by the dynamics of a Bose Josephson Junction (BJJ) [1 3]. The usefulness of squeezed states in improving phase sensitivity in interferometry has been demonstrated in a recent experiment [4]. An even better sensitivity is predicted to arise by employing macroscopic superpositions [5]. The presence of noise and of coupling with the environment causes decoherence and limits the experimental time for coherent manipulations. Decoherence may even prevent the production of certain entangled states, a fundamental issue in the quantum-to-classical transition [6]. It is thus important to study the robustness of these nonclassical states in the presence of noise. Several sources of decoherence in BJJ like particle losses [7], collisions with thermal atoms [8, 9] , interaction with the electromagnetic field [10], and random fluctuations of the trapping potential [11] have been identified and analyzed theoretically.

In this work we solve the quantum dynamics of a BJJ in the presence of a noise coupling linearly to the numberimbalance operator. This noise results from the fluctuations of the optical potential and of the magnetic field, which are, together with atom losses, the main sources of decoherence in the experiments of Ref. [4, 12, 13]. Our solution is exact and allows in particular to capture the decay of the Ramsey fringes visibility at short times (non-markovian regime). Furthermore, it shows that the macroscopic superpositions of phase states generated by the unitary dynamics are rather robust with respect to the noise considered. According to the usual scenario for decoherence [14, 15], by increasing the intensity of the noise these superpositions should be transformed into statistical mixtures of the same phase states at a noise intensity proportional to a negative power of the number of atoms, which characterizes here the "distance" between the phase states. For the aforementioned noise, we find that this is not the case. The typical noise intensity at which the coherences between the distinct phase states are lost is independent of the atom number and equal to (or even, for many-component superpositions, larger than) the noise intensity at which phase relaxation occurs. Phase relaxation means that each phase state of the superposition converges to a mixture of Fock states and acquires a completely undefined phase. At intermediate noise, the phase has spread significantly but some entanglement remains due to the non vanishing coherences among the phase states of the superposition. We quantify this entanglement by computing the quantum Fisher information [5] and estimate the gain in phase sensitivity with respect to separable states.

The paper is organized as follows. After introducing in Sec.II the two-mode approximation for the BJJ, we review in Sec.III the quenched dynamics of the junction in the absence of noise, leading to the formation of nonclassical states. Sec.IV presents our results on the effect of the noise on the density matrix of the atoms and on the visibility of the Ramsey fringes, while Sec.V analyzes the degradation of the coherence of macroscopic superpositions. Finally Sec.VI offers a summary and some concluding remarks.

\section{MODEL}

We describe the BJJ by a two-mode Hamiltonian [16]

$$
\hat{H}^{(0)}=\chi \hat{J}_{z}^{2}-\lambda \hat{J}_{z}-2 K \hat{J}_{x},
$$

where the angular momenta operators $\hat{J}_{x}, \hat{J}_{y}$, and $\hat{J}_{z}$ are related to the annihilation operator $\hat{a}_{j}$ of an atom in the mode $j=1,2$ by $\hat{J}_{x}=\left(\hat{a}_{1}^{\dagger} \hat{a}_{2}+\hat{a}_{2}^{\dagger} \hat{a}_{1}\right) / 2, \hat{J}_{y}=$ 
$-i\left(\hat{a}_{1}^{\dagger} \hat{a}_{2}-\hat{a}_{2}^{\dagger} \hat{a}_{1}\right) / 2$, and $\hat{J}_{z} \equiv \hat{n}=\left(\hat{a}_{1}^{\dagger} \hat{a}_{1}-\hat{a}_{2}^{\dagger} \hat{a}_{2}\right) / 2$, the latter being the number imbalance operator. We assume a fixed total number of atoms $N$, i.e. we do not account for atom losses. We take $N$ to be even for simplicity, the odd case being qualitatively similar. The Hamiltonian (11) models both a single-component Bose gas trapped in a double-well potential [12] - external Josephson junction and a binary mixture of atoms in distinct hyperfine states trapped in a single well [4, 17] - internal Josephson junction. In the external BJJ the two modes $i$ correspond to the lowest-energy spatial modes in each well. For the internal BJJ, the two relevant modes are the two hyperfine states. The first term in (1) describes the repulsive atomatom interactions; for the external BJJ, $\chi$ is the half of the sum of the interaction energies $U_{i}$ in the two modes, whereas for the internal BJJ $\chi=\left(U_{1}+U_{2}\right) / 2-U_{12}$ also depends on the inter-species interaction $U_{12}$. In both cases, $\lambda=\Delta E+(N-1)\left(U_{2}-U_{1}\right) / 2$ is related to the difference $\Delta E=E_{2}-E_{1}$ between the energies of the two modes. The last term in (11) corresponds to tunelling between the two wells or, in the internal BJJ, to a resonant laser field coupling the two hyperfine states. Both $\chi$ and $K$ are experimentally tunable parameters.

It is convenient to characterize a state $|\psi\rangle$ of the BJJ by its Husimi function $Q(\theta, \phi)=|\langle\theta, \phi \mid \psi\rangle|^{2}$ on the classical phase space (the Bloch sphere of radius $N / 2$ ), where

$$
|\theta, \phi\rangle=\sum_{n=-N / 2}^{N / 2}\left(\begin{array}{c}
N \\
n+\frac{N}{2}
\end{array}\right)^{1 / 2} \frac{\alpha^{n+N / 2}}{\left(1+|\alpha|^{2}\right)^{N / 2}}|n\rangle
$$

is a $\mathrm{SU}(2)$ coherent state [18], $\alpha \equiv \tan (\theta / 2) \exp (-i \phi)$, and $|n\rangle$ is the Fock state satisfying $\hat{J}_{z}|n\rangle=n|n\rangle$. For a coherent state (2) $Q$ is peaked around the vector $N(\sin \theta \cos \phi, \sin \theta \sin \phi,-\cos \theta) / 2$, the components of which are the expectation values of $\hat{J}_{x}, \hat{J}_{y}$, and $\hat{J}_{z}$ in this state. In particular for a phase state, i.e. a coherent state with $\theta=\pi / 2$, such a peak is located on the equator of the Bloch sphere. A Fock state has a $\phi$-independent distribution, with a peak in $\theta$ at $\theta=\arccos (-2 n / N)$.

\section{DYNAMICS IN THE ABSENCE OF NOISE}

In the absence of noise, let us describe the quenched dynamics of the BJJ induced by a sudden switch off of the Josephson coupling $K$ in (10) at time $t=0$. We take $\lambda=0$ for simplicity. Initially, the BJJ is in the phase state with $\phi=0$ (i.e., $\alpha=1$ ). This state is the ground state of the Hamiltonian (10) in the regime $K N \gg \chi$ where tunelling dominates interactions. In the internal BJJ, it can be produced by applying a short $\pi / 2$-pulse to the atoms initially in the lower level. Under the effect of the quench the phase starts diffusing along the equator of the Bloch sphere. The visibility of Ramsey fringes [19] at time $t>0$ reads [7]

$$
\nu^{(0)}(t) \equiv \frac{2}{N}\left\langle\hat{J}_{x}^{(0)}(t)\right\rangle=\cos ^{N-1}(\chi t) .
$$

At small times, the BJJ is in a squeezed state 20]. Later on, it returns to the initial state $|\alpha=1\rangle$ at the revival time $T \equiv 2 \pi / \chi$; at intermediate times $t_{q} \equiv T /(2 q)$ it is in a superposition $\left|\psi^{(0)}\left(t_{q}\right)\right\rangle=u_{0} \sum_{k=0}^{q-1} c_{k}\left|e^{-i 2 \pi k / q}\right\rangle$ of $q$ phase states [2, 3] with $\left|u_{0}\right|^{2}=1 / q$ and $c_{k}=e^{i \pi k(k+N) / q}$ (we have taken $q$ even). By (2), the matrix elements of the density matrix $\hat{\rho}^{(0)}\left(t_{q}\right)=\left|\psi^{(0)}\left(t_{q}\right)\right\rangle\left\langle\psi^{(0)}\left(t_{q}\right)\right|$ in the Fock basis are the sum over all $k, k^{\prime}=0, \cdots, q-1$ of

$$
\begin{aligned}
\left\langle n\left|\hat{\rho}_{k k^{\prime}}^{(0)}\left(t_{q}\right)\right| n^{\prime}\right\rangle & =\frac{1}{q} \frac{1}{2^{N}}\left(\begin{array}{c}
N \\
n+\frac{N}{2}
\end{array}\right)^{\frac{1}{2}}\left(\begin{array}{c}
N \\
n^{\prime}+\frac{N}{2}
\end{array}\right)^{\frac{1}{2}} \\
& \times e^{-2 i \pi\left(k n-k^{\prime} n^{\prime}\right) / q} e^{i \pi\left(k^{2}-k^{\prime 2}\right) / q}
\end{aligned}
$$

with $\hat{\rho}_{k k^{\prime}}^{(0)}\left(t_{q}\right)=q^{-1} c_{k} c_{k^{\prime}}^{*}\left|e^{-i 2 \pi k / q}\right\rangle\left\langle e^{-i 2 \pi k^{\prime} / q}\right|$. Since the dynamics does not couple the two modes, $\hat{J}_{z}$ is a constant of motion. Thus $\left\langle n\left|\hat{\rho}^{(0)}(t)\right| n\right\rangle$ is constant in time and equal to $P_{\alpha=1}(n)=2^{-N}\left(\begin{array}{c}N \\ n+N / 2\end{array}\right)$. In order to address later on the decoherence and phase relaxation of the superpositions of phase states, we decompose $\hat{\rho}^{(0)}\left(t_{q}\right)$ as

$$
\hat{\rho}^{(0)}\left(t_{q}\right)=\sum_{k=0}^{q-1} \hat{\rho}_{k k}^{(0)}\left(t_{q}\right)+\sum_{k \neq k^{\prime}=0}^{q-1} \hat{\rho}_{k k^{\prime}}^{(0)}\left(t_{q}\right) .
$$

The first sum in (5), which we will refer to as the "diagonal part" $\hat{\rho}_{\mathrm{d}}^{(0)}\left(t_{q}\right)$, is a statistical mixture of phase states. It is mainly responsible for the structure of the phase profile given by the Husimi distribution [21]. The second sum in (5), to be referred below as the "off-diagonal part" $\hat{\rho}_{\text {od }}^{(0)}\left(t_{q}\right)$, accounts for quantum correlations and interference effects, such as, for example, fringes in the eigenvalue probability distributions of $\hat{J}_{x}$ and $\hat{J}_{y}[21]$.

\section{DYNAMICS IN THE PRESENCE OF PHASE NOISE}

We now account for the effect of noise by considering the Hamiltonian

$$
\hat{H}(t)=\chi \hat{J}_{z}^{2}-\lambda(t) \hat{J}_{z}
$$

where $\lambda(t)$ is a classical stochastic process. Since $\left[\hat{H}(t), \hat{J}_{z}\right]=0$ at all times, $\hat{J}_{z}$ is conserved as in the noiseless case. Neglecting the fluctuations of $U_{i}$ (which seems justified in the experiments), the fluctuations of $\lambda$ are equal to those of $\Delta E$ and are independent of $N$. For a given realization of the process $\lambda$, the Schrödingerevolved state is obtained from the state $\left|\psi^{(0)}(t)\right\rangle=$ $e^{-i \chi \hat{J}_{z}^{2} t}|\alpha=1\rangle$ in the absence of noise through a rigid rotation around the $z$-axis by a random angle $\phi(t) \equiv$ 
$-\int_{0}^{t} d \tau \lambda(\tau)$, i.e., $|\psi(t)\rangle=e^{-i \phi(t) \hat{J}_{z}}\left|\psi^{(0)}(t)\right\rangle$. The phase $\phi$ has a distribution $f(\phi, t)=\int d P[\lambda] \delta(\phi(t)-\phi)$ where $P[\lambda]$ is the probability distribution of the process $\lambda$. Averaging over all realizations of $\lambda$ leads to the density matrix $\hat{\rho}(t)=\int d P[\lambda]|\psi(t)\rangle\langle\psi(t)|$. This is the analog of tracing out the bath degrees of freedom in models of systems coupled to quantum baths. We obtain

$$
\hat{\rho}(t)=\int_{-\infty}^{\infty} d \phi f(\phi, t) e^{-i \phi \hat{J}_{z}} \hat{\rho}^{(0)}(t) e^{i \phi \hat{J}_{z}}
$$

where $\hat{\rho}^{(0)}(t)=\left|\psi^{(0)}(t)\right\rangle\left\langle\psi^{(0)}(t)\right|$ is the density matrix in the absence of noise. By projecting Eq.(17) over the Fock basis we get

$$
\begin{aligned}
\left\langle n|\hat{\rho}(t)| n^{\prime}\right\rangle & =\int_{-\infty}^{\infty} d \phi f(\phi, t) e^{-i \phi\left(n-n^{\prime}\right)}\left\langle n\left|\hat{\rho}^{(0)}(t)\right| n^{\prime}\right\rangle \\
& =\tilde{f}\left(n^{\prime}-n, t\right)\left\langle n\left|\hat{\rho}^{(0)}(t)\right| n^{\prime}\right\rangle
\end{aligned}
$$

where $\tilde{f}(m, t)=\int_{-\infty}^{\infty} d \phi f(\phi, t) e^{i m \phi}=\overline{e^{i m \phi(t)}}$ is the Fourier transform of $f(\phi, t)$ with respect to $\phi$ and the overline denotes the average over the realizations of the noise $\lambda$ according to the probability distribution $P[\lambda]$. To be specific, let us consider a gaussian noise. Then $\tilde{f}(m, t)=e^{-a^{2}(t) m^{2} / 2} e^{-i \bar{\lambda} t m}$, where the variance $a^{2}(t)$ is given in terms of the noise correlation function $h\left(\tau-\tau^{\prime}\right)=\overline{\lambda(\tau) \lambda\left(\tau^{\prime}\right)}-\bar{\lambda}^{2}=\overline{\Delta E(\tau) \Delta E\left(\tau^{\prime}\right)}-\overline{\Delta E}^{2}$ by $a^{2}(t)=\int_{0}^{t} d \tau \int_{0}^{t} d \tau^{\prime} h\left(\tau-\tau^{\prime}\right)=2 \int_{0}^{t} d \tau \int_{0}^{\tau} d u h(u)$. (Note that $h$ depends on the time difference $\tau-\tau^{\prime}$ by the stationarity of the process, which also implies $\overline{\lambda(t)}=\overline{\lambda(0)} \equiv \bar{\lambda}$.) This yields

$$
\left\langle n|\hat{\rho}(t)| n^{\prime}\right\rangle=e^{-\frac{a^{2}(t)\left(n-n^{\prime}\right)^{2}}{2}} e^{i \bar{\lambda} t\left(n-n^{\prime}\right)}\left\langle n\left|\hat{\rho}^{(0)}(t)\right| n^{\prime}\right\rangle .
$$

The effect of the noise is to spread the noiseless evolution $\hat{\rho}^{(0)}(t)$ along the equator of the Bloch sphere by the amount $a(t)$; in this sense, it is a pure-dephasing noise. Since our result (9) does neither rely on a perturbative approach nor on a Markov approximation, it is valid also for strong noise and at short times. The variance $a^{2}(t)$ does not depend on $N$ and completely characterizes the effect of the noise on the BJJ. It is given by

$$
a^{2}(t) \simeq \begin{cases}h(0) t^{2} & \text { if } t \leq t_{c} \quad \text { (small time) } \\ 2 \int_{0}^{\infty} d \tau h(\tau) t & \text { if } t \gg T_{c} \text { (Markov) }\end{cases}
$$

where we have introduced the noise time scales $t_{c}$ and $T_{c}$, $t_{c}$ being the largest time such that $h(\tau) \simeq h(0)=\delta \lambda(0)^{2}$ for $|\tau| \leq t_{c}$ and $T_{c}$ the smallest time such that $h(\tau) \simeq 0$ for $\tau \geq T_{c}$. Eq. (9) shows how the noise suppresses the off-diagonal elements of the density matrix in the Fock basis. At long times $t \gg\left(\int_{0}^{\infty} d \tau h(\tau)\right)^{-1}$ the state of the BJJ converges to a statistical mixture of Fock states with the same probabilities as the initial state,

$$
\hat{\rho}(\infty)=\sum_{n=-N / 2}^{N / 2} P_{\alpha=1}(n)|n\rangle\left\langle n\left|=\int_{0}^{2 \pi} \frac{d \phi}{2 \pi}\right| \frac{\pi}{2}, \phi\right\rangle\left\langle\frac{\pi}{2}, \phi\right| .
$$
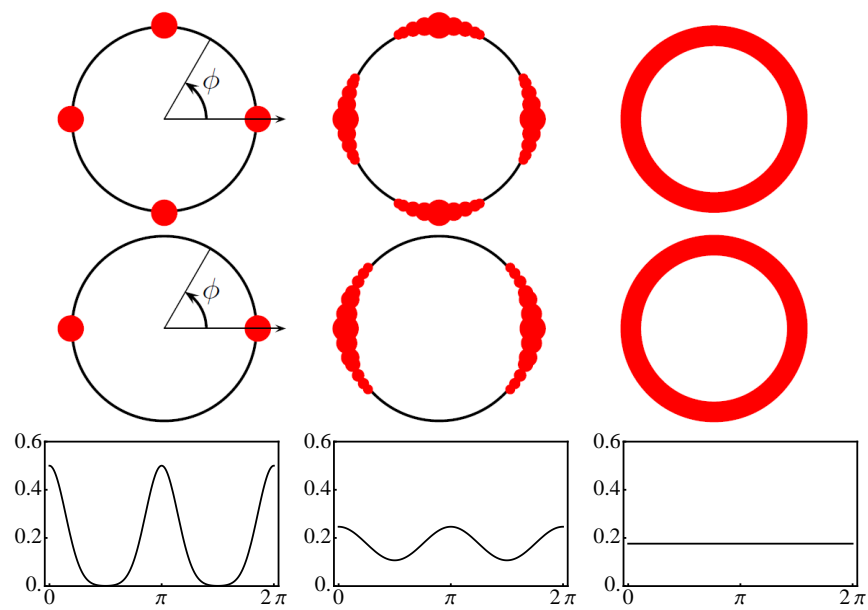

FIG. 1: (Color online) Phase relaxation of the $q=4$ and $q=2$ macroscopic superpositions in the presence of noise sketched along the equator $\theta=\pi / 2$ of the Bloch sphere. Top panels: $q=4\left(t_{4}=T / 8\right)$ and $a_{4}=0,0.64,2.05$ (from left to right). Middle panels: $q=2\left(t_{2}=T / 4\right)$ for the same noise intensities $\int_{0}^{\infty} d \tau h(\tau)$ in the Markov regime $\left(a_{2}=0,0.9,2.9\right)$. The circle sizes illustrate qualitatively the phase distribution $f\left(\phi, t_{2,4}\right)$. For intermediate noise (middle column), the superposition is closer to the steady state (last column) for $q=4$ than for $q=2$. Bottom panels: Husimi distribution $Q(\theta=\pi / 2, \phi)$ for $q=2$ for the same values of $a_{2}$. Here $\bar{\lambda}=0$ and $N=10$.

The last equality is obtained from (2). It means that at large times the phase $\phi$ is uniformly spread on $[0,2 \pi]$, as is the case for Fock states (Fig 1, right panels).

For a non-gaussian noise, the two exponentials in the right-hand side of (9) coincide with the cumulant expansion of $\tilde{f}(m, t)=\overline{e^{i m \phi(t)}}$ up to the second cumulant 22 and higher cumulants yields extra factors $e^{b_{p}(t)\left(n-n^{\prime}\right)^{p}}$, $p=3,4, \ldots$. For small times $t \leq t_{c}$, these factors are close to unity and the right-hand side of (9) still gives a good approximation of the matrix elements of $\hat{\rho}(t)$.

Under the effect of the noise, the visibility (3) acquires an additional decaying factor due to the above-mentioned phase spreading. Indeed, one easily obtains from (9)

$$
\nu(t)=\frac{2}{N} \operatorname{tr}\left[\hat{\rho}(t) \hat{J}_{x}\right]=e^{-\frac{a^{2}(t)}{2}} \cos (\bar{\lambda} t) \nu^{(0)}(t) .
$$

As found e.g. in superconducting circuits 23] and in quantum dots 24], the dephasing factor $e^{-a^{2}(t) / 2}$ displays a Gaussian decay at short times $t \leq t_{c}$, corresponding to the universal regime of Ref. [15], and an exponential decay at long times $t \gg T_{c}$ (Markov regime). A Gaussian decay of the visibility (12) has been observed experimentally in the internal BJJ at small values of the interactions $\chi$ [25]. This indicates that the experiment was performed in the small-time, non-markovian regime. The effect of the noise on the visibility decay in this regime is shown in Fig 2 for experimentally relevant parameters [25]. A direct comparison with the experiment requires to include in the model atom losses. 


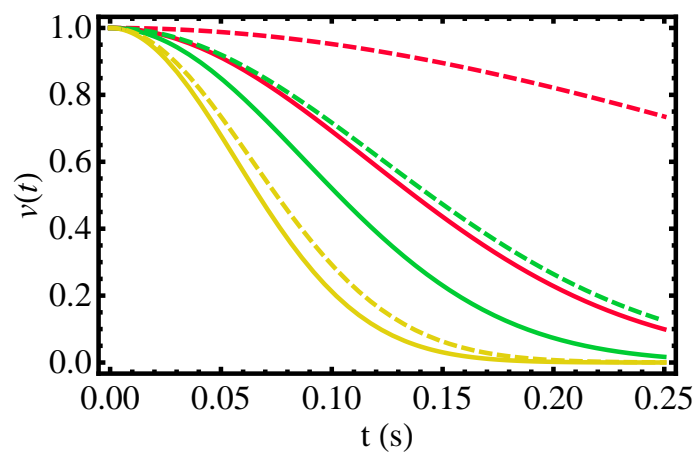

FIG. 2: (Color online) Visibility $\nu(t)$ as a function of time (in units of seconds) for $\chi=\pi \cdot 0.05 \mathrm{~Hz}, \pi \cdot 0.13 \mathrm{~Hz}, \pi \cdot 0.25 \mathrm{~Hz}$ (from top to bottom), $N=400$. Solid lines: decay of $\nu(t)$ in Eq. (12) in the limit $\chi t \ll 1$ and $\bar{\lambda} t \ll 1$ with $a^{2}(t)=h(0) t^{2}$ and $h(0)^{1 / 2}=8 \mathrm{~Hz}$. Dashed lines: decay of $\nu^{(0)}(t)$ under the unitary evolution only. For small values of the interactions the decay is mainly due to the noise.

\section{DECOHERENCE AND RELAXATION OF MACROSCOPIC SUPERPOSITIONS}

Let us then study the impact of decoherence and relaxation on a macroscopic superposition at the fixed time $t_{q}$ as a function of the noise intensity. We assume that $q$ is even. Using (9) and the decomposition (5) of the density matrix into diagonal and off-diagonal parts, and setting $a_{q} \equiv a\left(t_{q}\right)$, one gets

$$
\left\langle n\left|\hat{\rho}_{\mathrm{d}, \mathrm{od}}\left(t_{q}\right)\right| n^{\prime}\right\rangle=e^{-\frac{a_{q}^{2}\left(n-n^{\prime}\right)^{2}}{2}}\left\langle n\left|\hat{\rho}_{\mathrm{d}, \mathrm{od}}^{(0)}\left(t_{q}\right)\right| n^{\prime}\right\rangle
$$

up to a phase factor irrelevant for decoherence. Since the effect of the noise in Eq.(13) factorizes out let us concentrate on the structure of the density matrix in the absence of noise. By (4) and the definition following Eq. (5) we have $\left\langle n\left|\hat{\rho}_{\mathrm{d}}^{(0)}\left(t_{q}\right)\right| n^{\prime}\right\rangle \propto \sum_{k=0}^{q-1} e^{-\frac{2 i \pi}{q} k\left(n-n^{\prime}\right)}=0$ if $n^{\prime} \neq n$ modulo $q$ and

$$
\left\langle n\left|\hat{\rho}_{\mathrm{d}}^{(0)}\left(t_{q}\right)\right| n^{\prime}\right\rangle=\frac{1}{2^{N}}\left(\begin{array}{c}
N \\
n+\frac{N}{2}
\end{array}\right)^{1 / 2}\left(\begin{array}{c}
N \\
n^{\prime}+\frac{N}{2}
\end{array}\right)^{1 / 2}
$$

otherwise. Therefore

$$
\left\langle n\left|\hat{\rho}_{\mathrm{d}}^{(0)}\left(t_{q}\right)\right| n^{\prime}\right\rangle= \begin{cases}\left\langle n|\hat{\rho}(0)| n^{\prime}\right\rangle & \text { if } n^{\prime}=n \text { modulo } q \\ 0 & \text { if } n^{\prime} \neq n \text { modulo } q\end{cases}
$$

and

$\left\langle n\left|\hat{\rho}_{\mathrm{od}}^{(0)}\left(t_{q}\right)\right| n^{\prime}\right\rangle= \begin{cases}0 & \text { if } n^{\prime}=n \text { modulo } q \\ e^{i \frac{\pi}{q}\left(n^{\prime 2}-n^{2}\right)}\left\langle n|\hat{\rho}(0)| n^{\prime}\right\rangle & \text { if } n^{\prime} \neq n \text { modulo } q\end{cases}$

The last equality follows from (14) and from the identity $\hat{\rho}^{(0)}\left(t_{q}\right)=e^{-i t_{q} \chi \hat{J}_{z}^{2}} \hat{\rho}(0) e^{i t_{q} \chi \hat{J}_{z}^{2}}$. In particular, we get $\left\langle n\left|\hat{\rho}_{\mathrm{od}}^{(0)}\left(t_{q}\right)\right| n \pm 1\right\rangle \neq 0$ from (15) (since $q \geq 2$ is even).

Thus from (13)-15) we obtain that $\hat{\rho}_{\mathrm{d}}\left(t_{q}\right) \rightarrow \hat{\rho}(\infty)$ when $a_{q} q \gg 1$ (phase relaxation) and $\hat{\rho}_{\text {od }}\left(t_{q}\right) \rightarrow 0$ when
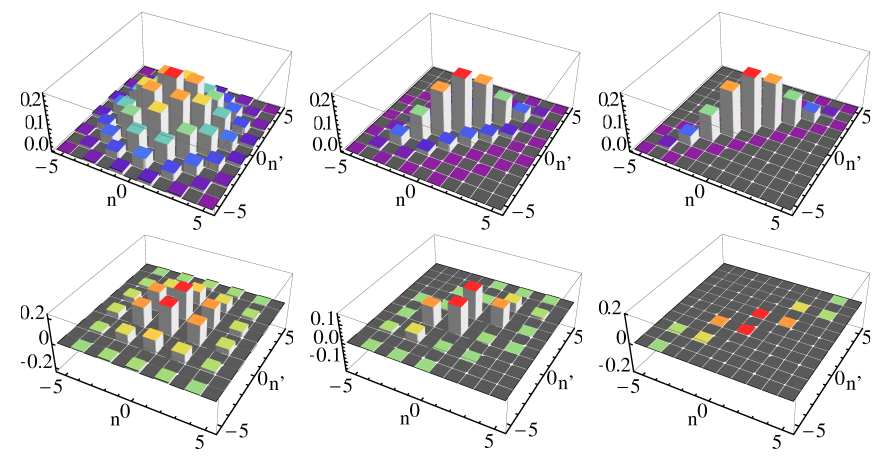

FIG. 3: (Color online) Top: relaxation of the diagonal part of the density matrix in the Fock basis (13) for $q=2$ and $N=10$ to the diagonal matrix (11) as the noise is increased from $a_{2}=0$ (left) to $a_{2}=0.9$ (middle) and $a_{2}=2.9$ (right panel). Bottom: off-diagonal part of the density matrix for $q=2$ and the same values of $a_{2}$, its vanishing indicates decoherence among the components of the macroscopic superposition.

$a_{q} \gg 1$ (decoherence). Hence, in the strong noise limit the diagonal part of $\hat{\rho}(t)$ relaxes to the steady state (11) and the off-diagonal part is washed away (see Fig[3, right panels). Remarkably, the decoherence factor in Eq. (13) does not depend on the atom number $N$. Also note the different noise scales relevant for decoherence, $a_{q}$, and phase relaxation, $a_{q} q$. When increasing the noise intensity, $\hat{\rho}_{\mathrm{d}}\left(t_{q}\right)$ approaches $\hat{\rho}(\infty)$ before $\hat{\rho}_{\mathrm{od}}\left(t_{q}\right)$ vanishes. The higher the number of components $q$ in the superposition, the more pronounced is this effect. In fact, superpositions with higher $q$ are better protected against decoherence since they are formed at shorter times and $a(t)$ increases with time. Moreover, in the Markov regime $t_{q} \gg T_{c}$, for a fixed noise intensity phase relaxation has a stronger effect on states with higher $q$, as illustrated in Fig. 1. Indeed, by (10), (13), and (14), the $n \neq n^{\prime}$ matrix elements of $\hat{\rho}_{\mathrm{d}}\left(t_{q}\right)$ are damped by a factor equal to or smaller than $e^{-a_{q}^{2} q^{2} / 2} \simeq \exp \left[-(\pi q / \chi) \int_{0}^{\infty} d \tau h(\tau)\right]$. In the small-time regime $t_{q} \leq t_{c}$, all the $q$-component superpositions relax to $\rho(\infty)$ at the same ( $q$-independant) noise intensity (since $a_{q} q$ is independent of $q$ ). As a consequence of the distinct noise scales for decoherence and phase relaxation, the BJJ does not turn into a statistical mixture of phase states but relaxes directly to the mixture of Fock states (11). This is illustrated in Fig 3 for the two-component superposition.

Phase relaxation can be represented by the Husimi distribution of $\hat{\rho}_{\mathrm{d}}\left(t_{q}\right)$ [21], which for $q=2, N \gg 1$, and $a_{2} \gg N^{-1 / 4}$ is given by $Q_{\mathrm{d}}(\theta, \phi)=\sum_{k=0}^{1} \int d \phi^{\prime} f\left(\phi^{\prime}, t_{2}\right)\left|\left\langle\theta, \phi \mid \frac{\pi}{2}, \phi^{\prime}+\pi k\right\rangle\right|^{2} / 2 \simeq$ $Q_{\infty}(\theta) \Theta_{3}\left(-\phi-\pi \bar{\lambda} /(2 \chi), e^{-2 a_{2}^{2}}\right)$ with $\Theta_{3}$ the Theta function [26] and $Q_{\infty}(\theta) \simeq\left(\frac{1+\sin \theta}{2}\right)^{N+1 / 2} / \sqrt{\pi N \sin \theta}$ the distribution of the state (11); $Q(\pi / 2, \phi)$ is plotted for various values of $a_{2}$ in Fig.1. In the absence of noise it shows peaks at $\phi=0$ and $\pi$, which correspond to the two coherent states of the superposition. The 
peaks are smeared at increasing $a_{2}$, and finally at $a_{2} \gg 1$ the Husimi distribution reaches the flat profile $Q(\pi / 2, \phi)=Q_{\infty}(\pi / 2)$.

An important consequence of the anomalous decoherence found in this model is that at intermediate noise strength associated to an already important phase relaxation, i.e., such that $\hat{\rho}_{\mathrm{d}}\left(t_{q}\right)$ is close to $\hat{\rho}(\infty)$ (e.g. for $a_{2}=$ 0.9 in Fig (3), the system still displays quantum correlations, which could be exploited in interferometry. These correlations can be quantified by computing the quantum Fisher information $F_{Q}[\hat{\rho}]$ associated to the $N$-particle density matrix $\hat{\rho}[5,27]$. The quantum Fisher information allows to estimate the best possible phase sensitivity $\Delta \theta$ in an interferometric scheme according to the generalized uncertainty principle $\Delta \theta \geq 1 /\left(\sqrt{m} \sqrt{F_{Q}[\hat{\rho}]}\right), m$ being the number of measurements performed [27]. For separable states we have $F_{Q}[\hat{\rho}] \leq N$ and $\Delta \theta \geq \Delta \theta_{\mathrm{SN}}=1 / \sqrt{m N}$. Sub-shot noise sensitivities $\Delta \theta<\Delta \theta_{\mathrm{SN}}$ can be achieved for states $\hat{\rho}$ satisfying $F_{Q}[\hat{\rho}]>N$, which is also a sufficient condition for multi-particle entanglement [5]. For $a_{2}=0$, the two-component superposition $\hat{\rho}^{(0)}\left(t_{2}\right)$ has $F_{Q}=N^{2}$ [28], corresponding to the Heisenberg limit $\Delta \theta=1 / \sqrt{m} N$. For noise strength $a_{2}=0.9$ and $N=10$ we find $[28,29] F_{Q}\left[\hat{\rho}\left(t_{2}\right)\right] \simeq 58$, which is indeed still larger than $F_{Q}\left[\hat{\rho}_{\mathrm{d}}^{(0)}\left(t_{2}\right)\right]=N$ and leads to a sensitivity gain $\Delta \theta / \Delta \theta_{S N}$ of $-3.8 \mathrm{db}$ with respect to the use of separable states. In the limit $a_{2} \gg 1, \hat{\rho}\left(t_{2}\right) \simeq \hat{\rho}(\infty)$ has a Fisher information $F_{Q}=N(N-1) /(2 N+2)<N$ [29].

\section{SUMMARY AND CONCLUDING REMARKS}

We have treated analytically the time evolution of a phase state driven simultaneously by the atomic interactions and by a noise coupled to the number imbalance operator $\hat{J}_{z}$. We have derived an exact expression for the density matrix, as well as for the noise-induced decay of the Ramsey visibility. The effect of the noise on the creation of macroscopic superpositions of phase states is to cause decoherence, i.e., the vanishing of quantum correlations. We have found that if the fluctuations of the atomic interactions are negligible, decoherence is "less efficient" than phase relaxation, especially for superpositions with a large number of components. As a consequence, the states generated by the noisy dynamics could in principle lead to sub-shot noise precision in interferometry.

The surprising fact that decoherence is not enhanced by increasing the atom number $N$ is specific to the noise considered. Indeed, such a noise is applied perpendicularly to the equator of the Bloch sphere where the phase states of the superpositions lay. As a result, the noise is insensitive to the separation between these states, which scales with $N$. However, such superpositions are very fragile under a noise applied parallel to the equatorial plane, which resolves the separation between the compo- nents. This yields an indication as to which classical noise to reduce to preserve the coherence in superpositions of the phase states: this is the noise in directions parallel to this plane. For example, stochastic fluctuations on the tunnel amplitude $K$ give rise to rapid decoherence of the macroscopic superposition $\left(|\alpha=1\rangle+e^{i \gamma}|\alpha=-1\rangle\right) / \sqrt{2}$ at a rate increasing with the atom number, without inducing relaxation.

\section{ACKNOWLEDGMENTS}

We thank C. Gross and M. Oberthaler for useful discussions on experimental issues and a careful reading of the manuscript. We acknowledge financial support from CNRS, the MIDAS project, and the project ANR-09BLAN-0098-01.

* Electronic address: giulia.ferrini@grenoble.cnrs.fr

[1] A. Smerzi et al, Phys. Rev. Lett. 79, 357 (1997).

[2] B. Yurke and D. Stoler, Phys. Rev. Lett. 57, 13 (1986); D. Stoler, Phys. Rev. D 4, 2309 (1971).

[3] G. Ferrini, A. Minguzzi and F.W.J. Hekking, Phys. Rev. A 78, 023606 (2008).

[4] C. Gross et al, Nature 464, 1165 (2010).

[5] L. Pezze and A. Smerzi, Phys. Rev. Lett. 102, 100401 (2009); P. Hyllus, O. Güne, and A. Smerzi, arXiv:0912.4349

[6] D. Giulini et al, Decoherence and the appearance of a Classical World in Quantum Theory (Springer, 1996).

[7] A. Sinatra and Y. Castin, Eur. Phys. J. D 4, 247 (1998).

[8] J. Anglin, Phys. Rev. Lett. 79, 6 (1997).

[9] D. Witthaut, F. Trimborn, and S. Wimberger, Phys. Rev. A 79, 033621 (2009).

[10] Y. P Huang and M.G. Moore, Phys. Rev. A 73, 023606 (2006).

[11] Y. Khodorkovsky, G. Kurizki, and A. Vardi, Phys. Rev. Lett. 100, 220403 (2008).

[12] J. Esteve et al, Nature 455, 1216 (2008).

[13] M.F. Riedel et al, Nature 464, 1170 (2010).

[14] M. Orszag, Quantum Optics, 2nd ed. (Springer, 2008)

[15] D. Braun, F. Haake, and W.T. Strunz, Phys. Rev. Lett. 86, 2913 (2001).

[16] G.J. Milburn et al, Phys. Rev. A 55, 4318 (1997).

[17] D.S. Hall et al, Phys. Rev. Lett. 81, 1539 (1998)

[18] W.M. Zhang, D.H. Feng and R. Gilmore, Rev. Mod. Phys. 62, 867 (1990).

[19] D.J. Wineland et al, Phys. Rev. A 50, 67 (1994).

[20] M. Kitagawa and M. Ueda, Phys. Rev. A 47, 5138 (1993).

[21] G. Ferrini, A. Minguzzi, and F.W.J. Hekking, Phys. Rev. A 80, 043628 (2009).

[22] N.G. Van Kampen, Stochastic Processes in Physics and Chemistry, Elsevier (Amsterdam, 2007).

[23] G. Ithier et al, Phys. Rev. B 72, 134519 (2005).

[24] A. Vagov et al., Phys. Rev. B 70, 201305 (2004).

[25] C. Gross, Ph.D. thesis, Heidelberg (2010), Sec. 4.5.

[26] M. Abramowitz and I.A. Stegun, Handbook of mathematical functions (Dover, 1972). 
[27] S.L. Braunstein and C.M. Caves, Phys. Rev. Lett. 72 3439 (1994); V. Giovannetti, S. Lloyd, and L. Maccone, Phys. Rev. Lett. 96, 010401 (2006)

[28] We compute the maximal Fisher information over all directions of angular momentum $\mathbf{J}$ as $F_{Q}=4 \lambda_{\max }$, where $\lambda_{\max }$ is the largest eigenvalue of the $3 \times 3$ ma- $\operatorname{trix} \Gamma_{i, j}=\sum_{l, m} \frac{\left(p_{l}-p_{m}\right)^{2}}{p_{l}+p_{m}}\left\langle l\left|\hat{J}_{i}\right| m\right\rangle\left\langle m\left|\hat{J}_{j}\right| l\right\rangle / 2$ and $|l\rangle$ the normalized eigenvectors of $\hat{\rho}$ with eigenvalues $p_{l}$, see [5].

[29] G. Ferrini, D. Spehner, A. Minguzzi, and F.W.J Hekking, in preparation. 